\title{
PREDICTION OF BUS TRAVEL TIME USING ARTIFICIAL NEURAL NETWORK
}

\author{
Johar Amita ${ }^{1}$, Jain Sukhvir Singh ${ }^{2}$, Garg Pradeep Kumar ${ }^{3}$ \\ 1,2,3 Centre for Transportation System, Indian Institute of Technology Roorkee, Roorkee 247667, \\ Uttarakhand, India
}

Received 21 May 2015; accepted 18 August 2015

\begin{abstract}
The objective of this study is to apply artificial neural network (ANN) for development of bus travel time prediction model. The bus travel time prediction model was developed to give real time bus arrival information to the passenger and transit agencies for applying proactive strategies. For development of ANN model, dwell time, delays and distance between the bus stops was taken as input data. Arrivals/departure times, delays, average speed between the bus stop and distance between the bus stops were collected for two urban routes in Delhi. Model was developed, validated and tested using GPS (Global Positioning System) data collected from field study. Comparative study reveals that ANN model outperformed the regression model in terms of accuracy and robustness.
\end{abstract}

Keywords: travel time, artificial neural network, regression model, buses, prediction.

\section{Introduction}

In metropolitan cities like Delhi, traffic congestion is the major problem encountered in our daily life which leads to decrease in accessibility and reliability. These problems are due to the growth of vehicles specially the private and intermediate transport service (Sinha, 2003; TRB, 2001; ECMT, 2002). Apart from metro rail, bus transit also plays a key role in urban transportation system in Delhi. Therefore, it is necessary to improve the quality of bus transit system.

The bus transport system in Delhi can be enhanced by providing the real time bus arrival information to the passenger through Advanced Public Transport System (APTS) (Gurmu and Fan, 2104). The availability of timely and accurate information of bus travel time is significant, as it attracts more commuters and increases their satisfaction (Jeong and Rilett, 2004). Therefore, to provide passenger with this type of information there is an urgent need to develop travel time prediction model with sufficient accuracy.

In this study an attempt has been made to develop a travel time prediction model for urban bus route in Delhi, India with the help of artificial neural network. Travel Time is an important component of transport system because it affects the efficiency of system and service attractiveness. If a travel time is appropriate it attracts more commuters along the route and increases the commuters' satisfaction. Travel time between any two stops $i$ and $i+1$ for a journey $j$ is defined as difference between the arrival time at stop $i+1$ and departure time at stop $i$ as shown in Eq. (1).

$T_{i-i+1}=T_{A i+1}-T_{D i}$

\footnotetext{
${ }^{1}$ Corresponding author: amitadce@iitr.ernet.in
} 
Where,

$\mathrm{T}_{i-i+1}=$ travel between stop $i$ and stop $i+1$ for journey $j$

$\mathrm{T}_{A i+1}=$ bus arrival time at stop $i+1$

$\mathrm{T}_{D i}=$ bus departure time

Organization of the paper is as follows: Section 2 covers the brief summary of past studies. Section 3 discuss about data collected through field studies. Section 4 provides the detail description of ANN. In Section 5 discuss the results for neural network training, validation and testing and also compare the ANN model results with regression model. In section 6 conclude with model discussion and recommending direction for future work.

\section{Research Survey}

Accurate prediction of bus travel time is important because it improves the quality of bus services. Accurate travel time information is essential as it attracts more commuters and increases commuter's satisfaction (Jeong and Rilett, 2004). A variety of prediction model has been developed in the past by various authors to predict bus travel time. The extensively used travel time prediction models were historical based models, regression models, kalman filter- based models, and artificial neural network models.

1. Historical Based Model: (Lin and Zeng, 1999; Williams and Hoel, 2003; Rice and Zwet, 2004). This type of model give the current and future travel time using historical travel time of prior journey based on the assumption that the traffic conditions remain stationary.
Lin and Zeng (1999) developed arrival time estimation algorithms based on historical data to provide real time information. The algorithms were developed with different assumption on input data, like schedule adherence, waiting time at time-check stop, bus location data and schedule information. They developed the algorithms for buses in rural area without considering the effect of traffic congestion and dwell time at bus stop. The performance of four algorithms was evaluated in terms of accuracy, steadiness and robustness, and at last the result shows that four algorithms show best performance.

2. Regression Models: (Abdelfattah and Khan, 1998; You and Kim, 2000; Zhang and Rice, 2003; Patnaik et al., 2004; Ramakrishna et al., 2006; Yu et al., 2011; Chang et al., 2010). Regression model estimates the values of dependent variables from the values of independent variables. Regression model can work under unstable traffic condition. Advantage of regression models is that they tell which inputs are less or more important for predicting travel time. Abdelfattah and Khan (1998) build a linear and nonlinear regression model to predict bus delay using simulation data. The model was used to predict bus delay under normal condition, when one traffic lane is blocked. It meets the calibration test and was confirmed by field data. Patnaik et al. (2004) build a regression model to calculate arrival time of the bus and also identify the problem that occurred during processing of data collected through APC (Automatic Passenger Counting) units. The independent variables used were distance between 
the stops, number of bus stops, number of passenger boarding and alighting, weather descriptor and dwell times. The model was used to calculate arrival time of bus under various conditions. They also identified that weather was an unimportant parameter in their model. Ramakrishna et al. (2006) builds a multiple linear regression model to predict bus travel time using GPS data. They also indentify that the parameters dwell stop at bus stops and intersection delay were very less correlated hence they were not considered for model development.

3. Kalman Filtering Models: (Chen and Chien, 2002; Cathey and Dailey, 2003; Chien and Kuchipudi, 2003; Shalaby and Farhan, 2004; Vanajakshi et al., 2008; Yu et al., 2010; Liu et al., 2012; Mazloumi et al., 2012; Jang, 2013; Gurmu and Fan, 2014). Kalman Filter is a recursive procedure that uses linear quadratic estimation model to estimates the future states of system. Shalaby and Farhan (2004) build a prediction model for bus arrival and departure time using Kalman filtering technique. The data was collected for 5 weekdays in the month of May with four buses which are equipped with GPS and APC (Automatic Passenger Counter).The Kalman filter technique is used for filter component and two algorithms were proposed for predictor component. Two Kalman filter algorithm was developed to estimate the running time and dwell time separately. Model was developed using four days of data, and validated using one day data. Hold out data and data from micro-simulation software VISSIM was used to check the performance of the proposed prediction model. The proposed model out performed than the other models (historical models, regression models and time lag recurrent neural models) in terms of accuracy.

4. Artifical Neural Network Models: (Smith and Demetsky, 1995; Dia, 2001; Chien et al., 2002; Tong and Hung, 2002; Mahmoudabadi, 2010; Zheng and Zuylen, 2013; Li and Chen, 2013). ANN model is used for solving nonlinear complex data related problem. The implementation of ANN model is done in two phases: learning phase and recalling phase. In the learning phase the model is trained and weights are assigned and the recalling phase used to apply the weights assigned during learning phase. Smith and Demetsky (1995) build a backpropagation model using neural networkbased technique to predict traffic volumes for intelligent vehicle highway systems. Chien et al. (2002) build a model using artificial neural network technique to predict the dynamic arrival time of bus. An adjustment factor was also developed to change predicted travel time with new input of real time data. CORSIM was used to simulate the data including volume and passenger demand. Finally, the reliability analysis for the proposed ANN will be evaluated by comparing the predicted and simulated arrival time at each stop.

\section{Data Collection}

The routes chosen for this study was bus route numbers 832 and 817 in Delhi, India. These routes were chosen to develop the travel time prediction model. These routes were chosen because these routes give direct connectivity to commercial and residential 
area to most of the commuters. The detail of both the routes are shown in Table 1.The urban bus route 832 runs from Janakpuri D Block to Inderlock Metro Station via Sagarpur, Tilak Nagar, Moti Nagar and
Inderlock while route number 817 runs from Kair Village to Inderlock Metro Station via kair Depot, Tuda Mandi, KakrolaMor, Janakpuri East Bus Stop, Tilak Nagar, Inderlock as shown in Fig. 1.

\section{Table 1}

Detail of Urban Bus Route Number 832 and 817

\begin{tabular}{|l|l|l|l|}
\hline \multirow{2}{*}{ S. No } & \multirow{2}{*}{ Detail of Bus Route } & Name of Urban Bus Route \\
\cline { 3 - 4 } & & Route No. 832 & Route No. 817 \\
\hline 1. & Length & $14(\mathrm{~km})$ & $28(\mathrm{~km})$ \\
\hline 2. & Travel Time & $60-80$ minutes & $100-120$ minutes \\
\hline 3. & Number of Bus Stops & 33 & 53 \\
\hline 4. & Number of Intersection & 16 & 20 \\
\hline
\end{tabular}

Data has been collected through site visit for the duration of 5 weekdays in the month of February, 2014. The data was collected separately for each route. For this study parameters were collected using handled GPS (Global Positioning System). Data collected include arrival time/departure times, delays, average speed between the bus stops and distance between the stops. Arrival /departure times at each stops was used to calculate the dwell time at each stop. Dwell time is an important component of travel time because it affects the quality of transit service. Dwell time at any stop $i$ for a journey $j$ is defined as difference between the departure time at stop $i$ and arrival time at stop $i$ as shown in Eq. (2).
$D T_{i}=T_{D i}-T_{A i}$

Where,

$\mathrm{DT}_{i}=$ bus dwell time at stop $i$

$\mathrm{T}_{A i}=$ bus arrival time at stop $i$

$\mathrm{T}_{D i}=$ bus departure time at stop $i$

The correlation among the variables has been shown in the Table 2. From Table 2 we found that speed is correlated with distance $(0.55)$ and delay (0.597) and hence it was dropped. Therefore, the input variables considered to develop the model were dwell time, delays and distance between the stops.

\section{Table 2}

Correlation of Variables

\begin{tabular}{|l|l|l|l|l|}
\hline Variable & Dwell Time & Distance & Delay & Speed \\
\hline Dwell Time & 1 & & & \\
\hline Distance & 0.1813837 & 1 & & \\
\hline Delay & -0.023431 & -0.16655 & 1 & \\
\hline Speed & 0.0428633 & 0.553016 & 0.597309925 & 1 \\
\hline
\end{tabular}




\section{Development of Model}

\subsection{Regression for Travel Time Prediction}

To develop the travel time prediction model, a multi-linear regression analysis was performed on the data collected for two urban bus routes. The independent variables used in the model include distance between the stops, delays (at intersection and due to congestion) and dwell time at stops. The dependent variable used is Travel Time (TT in seconds). Regression models were developed with similar data set that was used to develop ANN model. In regression analysis the total data set has been divided in the two parts that is training and testing in the ratio 75 and 25 percent respectively. After the model is developed 25 percent data was used to test the developed model. The finally calibrated model is as shown in Eq. (3).

$T T=a+b^{*}$ Distance $+c^{*}$ Delay $+d^{*}$ Dwell Time

Where, $a, b, c, d$ are constants.

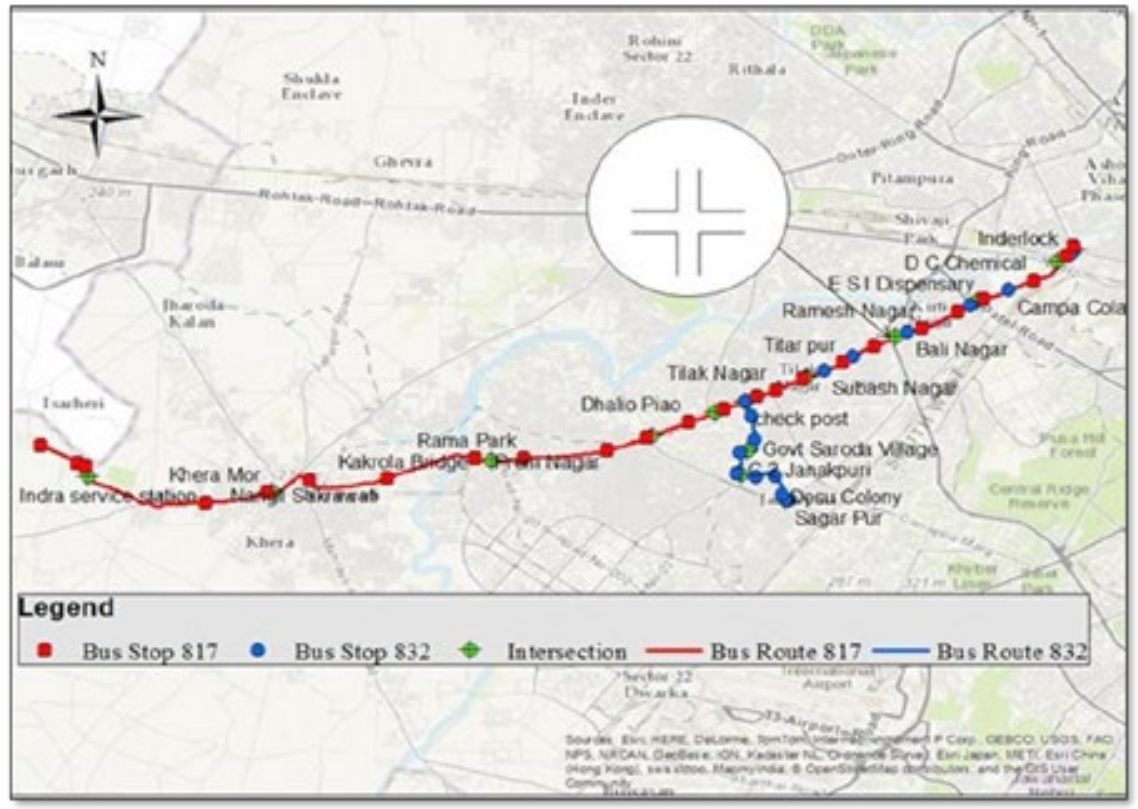

Fig. 1.

Layout of Bus Route No. 832 and 817

\subsection{ANN for Travel Time Prediction}

\subsubsection{Basic Background}

ANN is an information processing devices, which is comprised of large number of highly interconnected processing elements (PEs) that is inspired by the way biological nervous systems, such as brain process information (Hecht-Nielsen, 1987). In this information processing system, the elements are called neurons which process the information. The 
neuron with $n$ inputs calculates its output as shown in Eq. (4) (Demuth, 2007).

$a=f\left(\sum_{i=1}^{n} w_{i} p_{i}+b\right)$

Where,

$p_{i}$ is the value of $i^{\text {th }}$ input

$w_{i}$ is the value of $i^{\text {th }}$ weight

$b$ is the bias and $f$ is an activation function of the neuron

Various types of transfer functions are available that is step, logistic and hyperbolic tangent $(\tanh )$ function. The arrangement of neurons in to layers and patterns of connection within and in-between layer are generally called as architecture of net. Fig. 2 shows the architecture of ANN model. Architecture of ANN includes an input layer, a hidden layer and an output layer. In the input layer, the number of PEs is the same as the number of input variables that are used to forecast the required output. In output layer, the PEs determines the variables to be forecasted. The connection between the input and output layers are depending on the complexity of the problem one or several intermediate layers of PEs, generally called hidden layers. Depending on the complexity of the problem the number of hidden PEs within these layers is decided by trial and error approach.

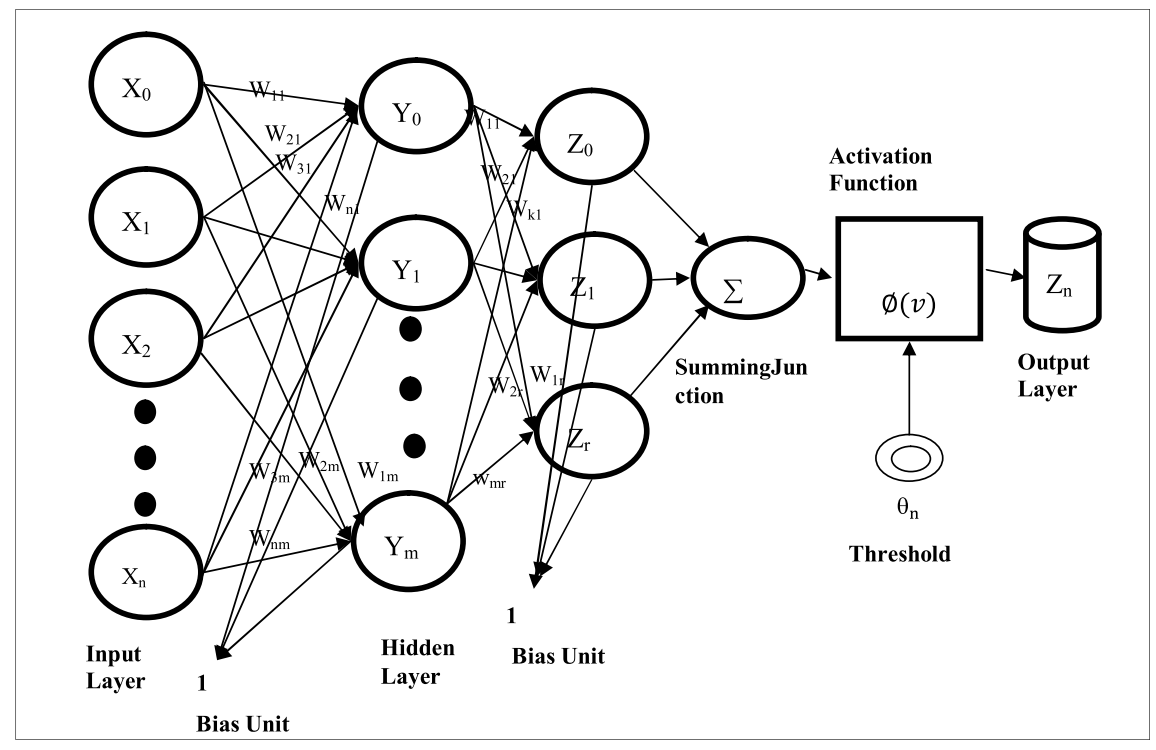

Fig. 2.

Structure of ANN

Depending upon the connections between PEs of different layers, various type network architectures are obtained. The processing elements are connected to each other by direct communication links, which is associated with weights. By adopting the weights of the communication links the ANN is supposed to learn correlation between 
input and output. To perform the training process large number of algorithms has been developed (Neural Ware, 1993). In this study the frequently used "backpropagation" network was implemented.

The training algorithm of back-propagation neural network involves four stages (Sivanandam et al., 2010):

1. Initialization of weight,

2. Feed Forward,

3. Back-propagation of error signals,

4. Updation of the weights and biases.

At firsts stage that is initialization of weights is used to initialize the weights to small random values. At second stage that is feed forward, each input unit receives an input signal and transmit it to output unit through hidden unit. If the output unit does not produce the desired output then third stage of back propagation of error signal is used in which error is propagated back to all the units in the previous layer. At the last stage, the weights and biases are updated in accordance with error signal. With these steps performing iteratively, the error can be minimized between network output and desired output using a predefined learning rule (Sivanandam et al., 2010).The transfer functions (i.e. activation function) are needed to establish nonlinearity into the network. For back-propagation, learning transfer function must be differentiable and bounded. The development procedure is completed once the network is trained.

The algorithm works best when the network inputs and outputs are normalized roughly in the range $[-1,1]$ (Demuth et al., 2007). For normalizing the input and output values according to the range Eq. (5) was used as shown below:

$$
X^{s}=2\left(X-X_{\text {min }}\right) /\left(X_{\text {max }}-X_{\text {min }}\right)-1
$$

Where $X^{s}$ gives the normalised value, $X$ is the original value, $X_{\min }$ and $X_{\text {max }}$ are the minimum and maximum value of $X$.

\subsubsection{Building Neural Network}

ANN has been applied for a wide variety of transportation problems and is relatively easy to use. Neural network automatically discover the relationship between the variables and naturally the fitting take place. Generally the network architecture is the single place where intuition of researchers comes into play. In ANN modelling based on the problem once can choose the required number of variables as there is no limit on the number of variable. ANN provides flexibility, massive parallelism, learning and generalization ability, accuracy and some amount of fault tolerance in prediction of travel time. For design of neural network there is no general theory or method. Mostly trial and error approach is used. During design of neural network the complexity arises while modelling of non-linear problem. Architecture of network, number of input variables, choosing of training algorithm and activation function are the basic features which must be considered in the design of neural network. All these are problem dependent quantities.

A set of data records considered for analysis of models are shown in Table 3. Whole database has been divided in to three parts training, validation and testing in the ratio 65,15 and 25 percentage respectively. In this study Model III was trained nine times using same set of training data (614 data records) and different number of neurons. Similarly, the model VI was trained nine times using same set of 
training data (979 data records) and different number of neurons. Dwell time, delays and distance between the stops were considered for development of models. Validation and testing data was used to compare the performance of ANN models. The performance measures MSE (Mean
Square Error) and coefficient of correlation $\left(\mathrm{R}^{2}\right)$ were used to estimate the prediction results. Table 4 and Table 5 give the detail of network with different structure for Model III and Model IV. From Table 4 and Table 5 it was found that network structure $(3,5$, $1)$ and $(3,15,1)$ produces best prediction.

Table 3

Data Records Considered for Analysis of Models

\begin{tabular}{|l|l|l|l|}
\hline S. No & Models Name & Data used for Development of Models & Set of Data Records \\
\hline 1. & Model I & 832 (upstream) & 512 \\
\hline 2. & Model II & 832 (downstream) & 512 \\
\hline 3. & Model III & 832 (combined upstream and downstream) & 1024 \\
\hline 4. & Model IV & 817 (upstream) & 832 \\
\hline 5. & Model V & 817 (downstream) & 800 \\
\hline 6. & Model VI & 817 (combined upstream and downstream) & 1632 \\
\hline
\end{tabular}

\section{Table 4}

Detail of Network with Different Structure for Model III

\begin{tabular}{|l|l|l|l|l|l|l|l|l|l|}
\hline Trial No & Train 1 & Train 2 & Train 3 & Train 4 & Train 5 & Train 6 & Train 7 & Train 8 & Train 9 \\
\hline No. of hidden layers & 1 & 1 & 1 & 1 & 1 & 1 & 1 & 1 & 1 \\
\hline No. of hidden neurons & 3 & 4 & 5 & 6 & 7 & 9 & 11 & 12 & 15 \\
\hline Training Function & Trainlm & Trainlm & Trainlm & Trainlm & Trainlm & Trainlm & Trainlm & Trainlm & Trainlm \\
\hline Transfer Function & Tansig & Tansig & Tansig & Tansig & Tansig & Tansig & Tansig & Tansig & Tansig \\
\hline Number of epochs & 1000 & 1000 & 1000 & 1000 & 1000 & 1000 & 1000 & 1000 & 1000 \\
\hline MSE (T) & $5.30 \mathrm{E}-2$ & $4.85-2$ & $4.09 \mathrm{E}-2$ & $4.83 \mathrm{E}-2$ & $4.96 \mathrm{E}-2$ & $4.83 \mathrm{E}-2$ & $5.67 \mathrm{E}-2$ & $4.95 \mathrm{E}-2$ & $4.91 \mathrm{E}-2$ \\
\hline MSE (V) & $5.83 \mathrm{E}-2$ & $5.73 \mathrm{E}-2$ & $4.61 \mathrm{E}-2$ & $5.84 \mathrm{E}-2$ & $5.96 \mathrm{E}-2$ & $6.31 \mathrm{E}-2$ & $5.37 \mathrm{E}-2$ & $4.77 \mathrm{E}-2$ & $5.41 \mathrm{E}-2$ \\
\hline MSE (Y) & $5.56 \mathrm{E}-2$ & $5.48 \mathrm{E}-2$ & $4.24 \mathrm{E}-2$ & $5.75 \mathrm{E}-2$ & $5.38 \mathrm{E}-2$ & $5.58 \mathrm{E}-2$ & $5.93 \mathrm{E}-2$ & $5.66 \mathrm{E}-2$ & $6.34 \mathrm{E}-2$ \\
\hline R(Y) & 0.8309 & 0.8480 & 0.8612 & 0.8458 & 0.8219 & 0.8505 & 0.8400 & 0.8521 & 0.8362 \\
\hline
\end{tabular}

$T=$ Training phase, $V=$ Validation Phase, $Y=$ Testing Phase 


\section{Table 5}

Detail of Network with Different Structure for Model VI

\begin{tabular}{|l|l|l|l|l|l|l|l|l|l|}
\hline Trial No & Train 1 & Train 2 & Train 3 & Train 4 & Train 5 & Train 6 & Train 7 & Train 8 & Train 9 \\
\hline No. of hidden layers & 1 & 1 & 1 & 1 & 1 & 1 & 1 & 1 & 1 \\
\hline No. of hidden neurons & 3 & 4 & 5 & 6 & 7 & 9 & 11 & 12 & 15 \\
\hline Training Function & Trainlm & Trainlm & Trainlm & Trainlm & Trainlm & Trainlm & Trainlm & Trainlm & Trainlm \\
\hline Transfer Function & Tansig & Tansig & Tansig & Tansig & Tansig & Tansig & Tansig & Tansig & Tansig \\
\hline Number of epochs & 1000 & 1000 & 1000 & 1000 & 1000 & 1000 & 1000 & 1000 & 1000 \\
\hline MSE (T) & $2.62 \mathrm{E}-3$ & $3.43 \mathrm{E}-3$ & $2.07 \mathrm{E}-3$ & $2.19 \mathrm{E}-3$ & $2.27 \mathrm{E}-3$ & $2.79 \mathrm{E}-3$ & $1.83 \mathrm{E}-3$ & $2.02 \mathrm{E}-3$ & $1.92 \mathrm{E}-3$ \\
\hline MSE (V) & $1.56 \mathrm{E}-3$ & $4.92 \mathrm{E}-3$ & $2.63 \mathrm{E}-3$ & $2.42 \mathrm{E}-3$ & $2.56 \mathrm{E}-3$ & $1.78 \mathrm{E}-3$ & $2.09 \mathrm{E}-3$ & $1.08 \mathrm{E}-3$ & $2.12 \mathrm{E}-3$ \\
\hline MSE (Y) & $7.34 \mathrm{E}-3$ & $3.05 \mathrm{E}-3$ & $3.09 \mathrm{E}-3$ & $3.03 \mathrm{E}-3$ & $1.95 \mathrm{E}-3$ & $3.55 \mathrm{E}-3$ & $5.28 \mathrm{E}-3$ & $4.50 \mathrm{E}-3$ & $2.09 \mathrm{E}-3$ \\
\hline R(Y) & 0.9219 & 0.9232 & 0.9329 & 0.9251 & 0.9330 & 0.9349 & 0.9042 & 0.9065 & 0.9357 \\
\hline
\end{tabular}

After the number of hidden neurons that produces best prediction for Model III and Model VI has been found. Then, Model I and Model II was trained using 307 set of training data and five numbers of hidden neurons (that produces best prediction for Model III). Similarly, the Model IV and Model V was trained using 499 and 480 set of training data and fifteen number of hidden neurons (that produces best prediction for Model VI).
The detail of the network structure for all the six models has been illustrated in Table 6. Based on the performance measure that is MSE and $\mathrm{R}^{2}$ it was found that Model I and Model II gives best prediction than Model III. Similarly, Model IV and Model V give best prediction than Model VI. Thus, it was found the separate model for route upstream and downstream gives best prediction that combined model. This was due to large number of disturbance in the combined model.

Table 6

Detail of Network Structure for all Six Models

\begin{tabular}{|l|l|l|l|l|l|l|l|l|l|}
\hline Model Name & $\begin{array}{l}\text { No. of } \\
\text { hidden } \\
\text { layers }\end{array}$ & $\begin{array}{l}\text { No. of } \\
\text { hidden } \\
\text { neurons }\end{array}$ & $\begin{array}{l}\text { Training } \\
\text { Function }\end{array}$ & $\begin{array}{l}\text { Transfer } \\
\text { Function }\end{array}$ & $\begin{array}{l}\text { Number } \\
\text { of epochs }\end{array}$ & MSE (T) & MSE (V) & MSE $(Y)$ & $R(Y)$ \\
\hline Model I & 1 & 5 & Trainlm & Tansig & 1000 & $1.85 \mathrm{E}-2$ & $2.29 \mathrm{E}-2$ & $2.09 \mathrm{E}-2$ & 0.9290 \\
\hline Model II & 1 & 5 & Trainlm & Tansig & 1000 & $6.34 \mathrm{E}-3$ & $5.45 \mathrm{E}-2$ & $7.59 \mathrm{E}-3$ & 0.9612 \\
\hline Model III & 1 & 5 & Trainlm & Tansig & 1000 & $4.09 \mathrm{E}-2$ & $4.61 \mathrm{E}-2$ & $4.24 \mathrm{E}-2$ & 0.8612 \\
\hline Model IV & 1 & 15 & Trainlm & Tansig & 1000 & $2.03 \mathrm{E}-4$ & $1.78 \mathrm{E}-3$ & $1.87 \mathrm{E}-3$ & 0.9616 \\
\hline Model V & 1 & 15 & Trainlm & Tansig & 1000 & $9.12 \mathrm{E}-4$ & $1.52 \mathrm{E}-3$ & $1.81 \mathrm{E}-3$ & 0.9713 \\
\hline Model VI & 1 & 15 & Trainlm & Tansig & 1000 & $1.92 \mathrm{E}-3$ & $2.12 \mathrm{E}-3$ & $2.09 \mathrm{E}-3$ & 0.9357 \\
\hline
\end{tabular}




\section{Results and Discussion}

Fig. 3 shows the graph between the Actual and Predicted travel time of four models for testing phase. The actual and predicted travel time was compared to check the validity of ANN models. Fig. 4 shows the scatter plots for models validation.

The performances of the developed models were evaluated by applying paired t-test. The paired t-test values of the models at 5\% level of significance are shown in the Table 7. Since the calculated $t$-value is less than the tabulated $t$-value for the models developed separately for each route, therefore using null hypothesis it was concluded that here was no major variation among the actual and predicted values. Hence, the models developed separately for each route are more suitable.

In this study, the result of the developed ANN models was compare with multiple regression models to check the accuracy and robustness of the models developed separately for each route. Three measure of effectiveness were calculated for all the four models: (1) RMSE (Root Mean Square Error) (2) MAPE (Mean Absolute Percentage Error) (3) $R^{2}$ (coefficient of correlation). RMSE is defined as the difference between the predicted and actual travel time. MAPE is defined as the average difference between the predicted and actual travel time. MAPE and RMSE have been defined as shown in the Eq. (6) and (7).

$$
\begin{aligned}
& M A P E=\frac{1}{n} \sum_{i=1}^{n}\left|\frac{\mathrm{y}_{1}-\mathrm{y}_{0}}{\mathrm{y}_{0}}\right| \times 100 \% \\
& R M S E=\sqrt{\frac{1}{n} \sum_{i=1}^{n}\left(y_{1}-y_{0}\right)^{2}}
\end{aligned}
$$

Where,

$y_{1}=$ is the predicted values of transit travel time

$y_{0}=$ is actual values of transit travel time

$n=$ is the number of data point in the set 


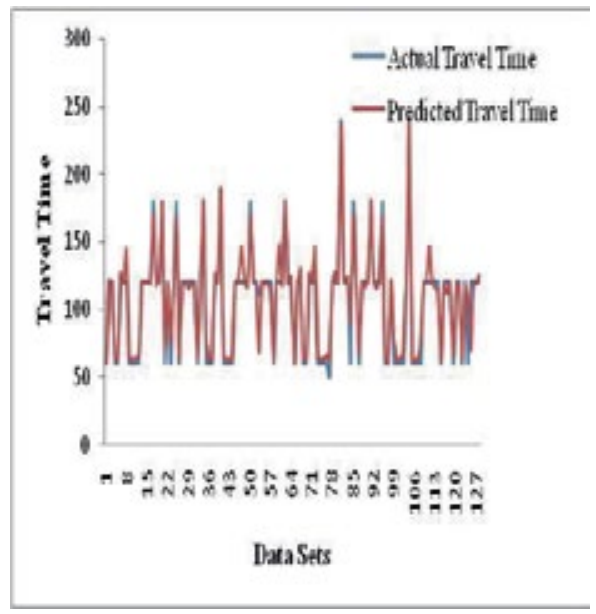

(a)

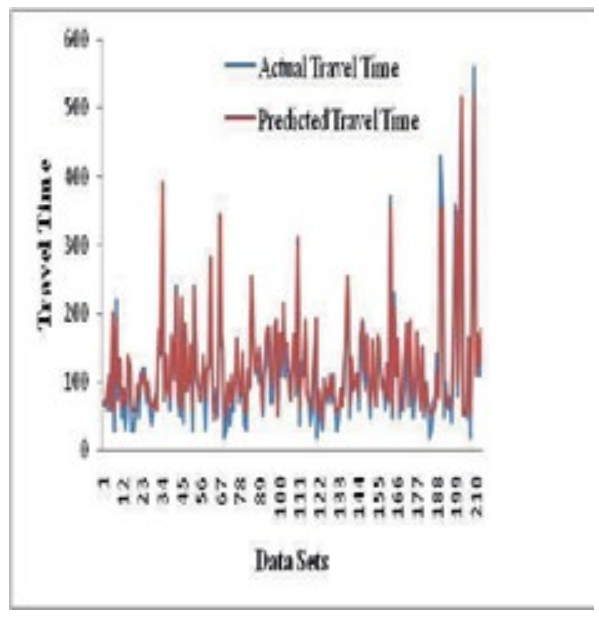

(c)

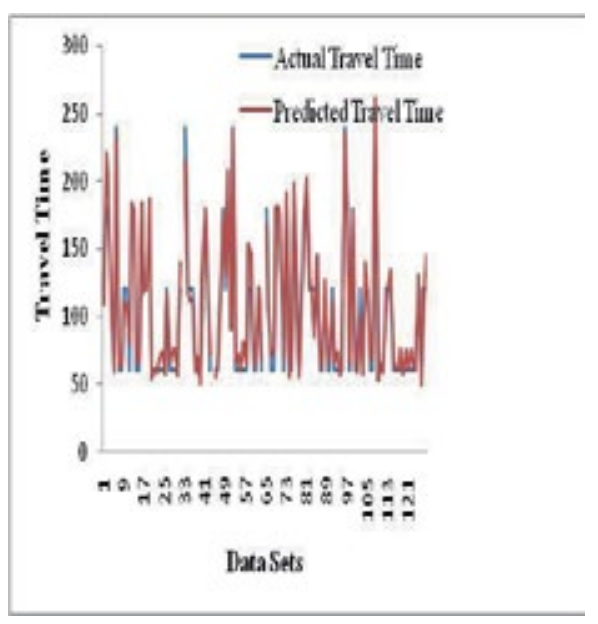

(b)

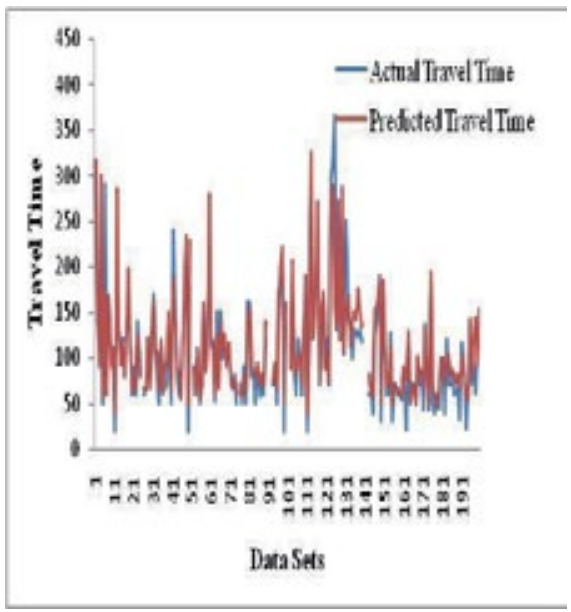

(d)

Fig. 3.

Actual and Predicted Travel Time of Four Models for Testing Phase

(a) Model I (b) Model II (c) Model IV (d) Model V 


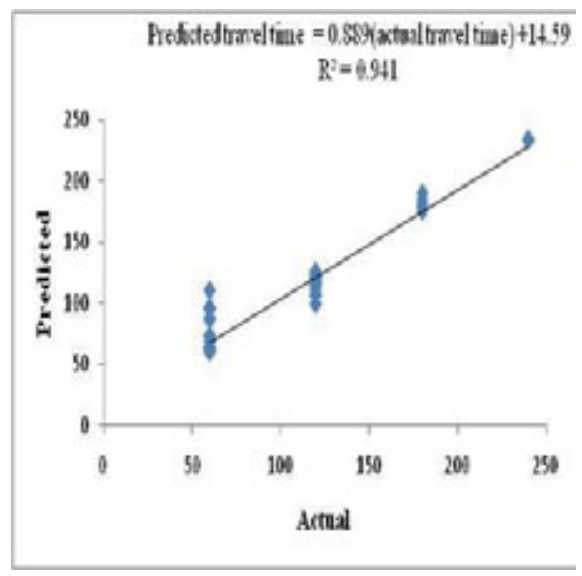

(a)

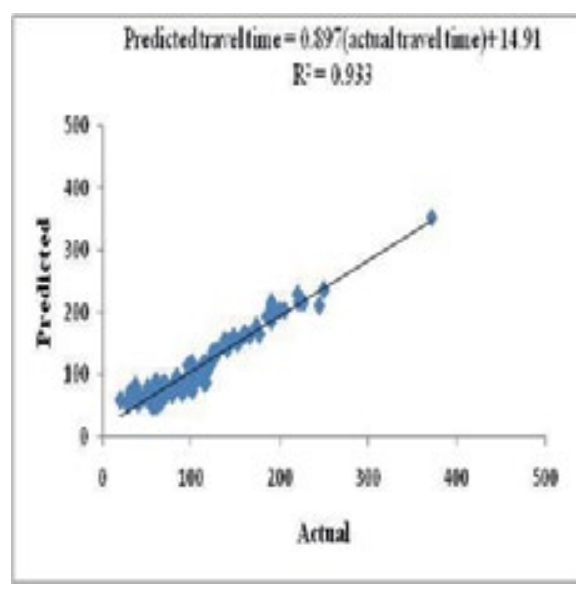

(c)

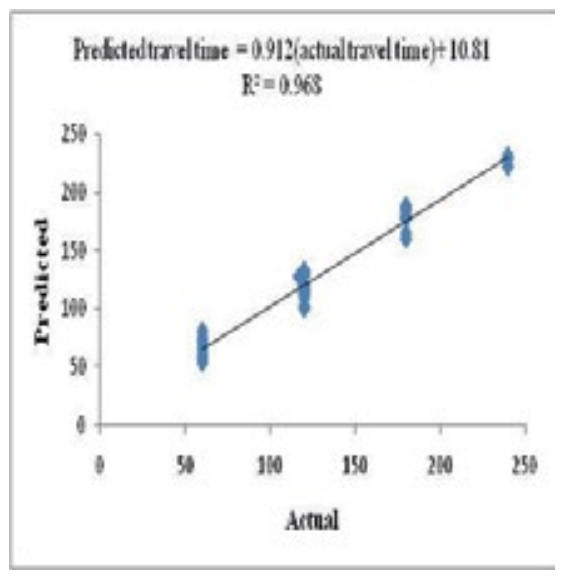

(b)

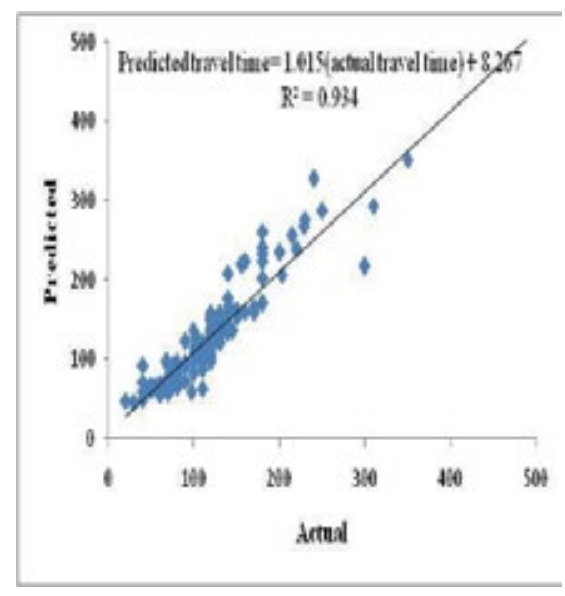

(d)

Fig. 4.

Scatter Plot between Actual and Predicted Travel Time of Four Models for Validation Phase (a) Model I (b) Model II (c) Model IV (d) Model V

Three measure of effectiveness that is RMSE, MAPE and $R^{2}$ for regression and $A N N$ models are shown in the Table 8. Results in the Table 8 shows that ANN models depict greater accuracy and robustness with less error as compared to regression models.
During training process ANN learns from examples and thus weights are adjusted accordingly to use this information during testing and cross validation. As compared to analytical and statistical model, ANN model generally produce better results 
with minimization of errors. Apart from good results and less error as compared to regression model, still there are some problems related to ANN modelling. In ANN modelling it is not possible to find the effect of each individual variable independently.

Bus travel time depends on multiple factors such as distance between stops, dwell time, delays, speed, arrival/departure time and schedule adherence. All these factors make travel time modelling a difficult and non linear problem. In a particular case study it is not possible to consider all the factors because of technical problem related with data collection. In this study, schedule adherence has not been taken into consideration.

Table 7

Summary of Output of Four Models for Testing Phase

\begin{tabular}{|l|l|l|l|l|l|l|l|}
\hline Models Name & $R 2$ & $R M S E$ & MAPE & Standard Deviation & t-test calculated & t-test tabulated & DOF \\
\hline Model I & 0.9290 & 10.432 & 6.527 & 10.268 & -2.263 & 1.9788 & 127 \\
\hline Model II & 0.9612 & 13.233 & 10.552 & 13.007 & -2.341 & 1.9788 & 127 \\
\hline Model IV & 0.9616 & 26.368 & 24.423 & 24.592 & -5.147 & 1.9719 & 207 \\
\hline Model V & 0.9713 & 32.830 & 20.573 & 32.721 & -1.527 & 1.9720 & 199 \\
\hline
\end{tabular}

RMSE $=$ Root Mean Square Error, MAPE = Mean Absolute Percentage Error, DOF = Degree Of Freedom

\section{Table 8}

Comparison between Regression and ANN Model for Testing Phase

\begin{tabular}{|l|l|l|l|l|l|l|}
\hline \multicolumn{2}{|l|}{ Model Name } & Equation & $R 2$ & $R M S E$ & MAPE & Standard Deviation \\
\hline \multirow{4}{*}{ Regression } & Model I & $0.937 \mathrm{x}+11.42$ & 0.812 & 18.427 & 16.234 & 17.874 \\
\cline { 2 - 7 } & Model II & $0.974 \mathrm{x}+8.545$ & 0.889 & 23.51 & 18.940 & 22.876 \\
\cline { 2 - 7 } & Model IV & $1.059 \mathrm{x}+0.588$ & 0.862 & 34.750 & 25.193 & 34.041 \\
\cline { 2 - 7 } & Model V & $1.121 \mathrm{x}-12.96$ & 0.881 & 46.692 & 22.054 & 46.743 \\
\hline \multirow{4}{*}{ ANN } & Model I & $0.914 \mathrm{X}+11.17$ & 0.9290 & 10.432 & 6.527 & 10.268 \\
\cline { 2 - 7 } & Model II & $0.954 \mathrm{x}+7.678$ & 0.9612 & 13.233 & 10.552 & 13.007 \\
\cline { 2 - 7 } & Model IV & $0.952 \mathrm{x}+7.767$ & 0.9616 & 26.368 & 24.423 & 24.592 \\
\cline { 2 - 7 } & Model V & $0.962 \mathrm{x}+6.876$ & 0.9713 & 32.830 & 20.573 & 32.721 \\
\hline
\end{tabular}

\section{Conclusion}

In the present study, ANN model has been developed to predict bus travel time for two selected urban bus route in Delhi. The performance of the model is evaluated using coefficient of correlation $\left(\mathrm{R}^{2}\right)$, root mean square error (RMSE), mean absolute percentage error (MAPE), standard deviation and t-test. Results shows that ANN model gives better results than regression model. For further work it is suggested that prediction model can be improved by incorporating the information of schedule adherence. It is also suggested to use Kalman Filter algorithm to predict travel time and compare the results with ANN model. 


\section{References}

Abdelfattah, A.M.; Khan, A.M. 1998. Models for predicting bus delays, Transportation Research Record. DOI: http://dx.doi.org/10.3141/1623-02, 1623(1): 8-15.

Cathey, F.W.; Dailey, D.J. 2003. A prescription for Transit Arrival/Departure Prediction using Automatic Vehicle Location Data, Transportation Research Part C: Emerging Technologies. DOI: http://dx.doi.org/10.1016/ S0968-090X(03)00023-8, 11: 241-264.

Chang, H.; Park, D.; Lee, S.; Lee, H.; Baek, S. 2010. Dynamic Multi-Interval Bus Travel Time Prediction Using Bus Transit Data, Transportmetrica, 6(1): 19-38.

Chen, M.; Chien, S. 2002. Dynamic Freeway Travel Time Prediction Using Probe Vehicle Data: Link-based vs. Path-based, Journal of the Transportation Research Board. DOI: http://dx.doi.org/10.3141/1768-19, 1768: 157-161.

Chien, S.I-J.; Kuchipudi, C.M. 2003. Dynamic Travel Time Prediction with Real-time and Historic Data, Journal of Transportation Engineering, ASCE. DOI: http://dx.doi.org/10.1061/(ASCE)0733947X(2003)129:6(608), 129(6): 608-616.

Chien, S.I-J.; Ding, Y.; Wei, C. 2002. Dynamic Bus Arrival Time Prediction with Artificial Neural Networks, Journal of Transportation Engineering. DOI: http://dx.doi.org/10.1061/ (ASCE)0733-947X(2002)128:5(429), 128(5): 429-438.

Demuth, H.; Beal, M.; Hagan, M. 2007. Neural Network Toolbox 5 user's Guide of the MATLAB Software. Natick, Mass.

Dia, H. 2001. An object-oriented Neural Network Approach to Short-term Traffic Forecasting, European Journal of Operational Research. DOI: http://dx.doi. org/10.1016/S0377-2217(00)00125-9, 131(2): 253-261.

European Conference on Ministers of Transport (ECMT). 2002. Implementing Sustainable Urban Transport Policies. Final Report, ECMT/OECD Publication Service, Paris, France.
Gurmu, K.Z.; Fan, W. 2014. Artificial Neural Network Travel Time Prediction Model for Buses using only GPS Data, Journal of Public Transportation, 17(2): 45-65.

Hecht-Nielsen, R. 1987. Kolmogorov's Mapping Neural Network Existence Theorem. In Proceedings of the First IEEE International Conference on Neural Networks, San Diego, 4-11.

Jang, J. 2013. Short-Term Travel Time Prediction using Kalman Filter Combined with a variable Aggregation Interval Scheme. In Proceeding of the Eastern Asian Society for Transportation Studies, 9.

Jeong, R.; Rilett, L.R. 2004. Bus Arrival Time Prediction Using Artificial Neural Network Model. In Proceedings of the IEEE Intelligent Transportation Systems Conference, Washington, D.C., USA, 988-993.

Li, C-S.; Chen, M-C. 2013. Identifying Important Variables for Predicting Travel Time of Freeway with Non-recurrent Congestion with Neural Network, Neural Computing and Applications. DOI: http://dx.doi. org/10.1007/s00521-012-1114-z, 23(6): 1611-1629.

Lin, W.H.; Zeng, J. 1999. Experimental Study of RealTime Bus Arrival Time Prediction with GPS Data, Transportation Research Record, 1666: 101-109.

Liu, X.; Chien, S.I.; Chen, M. 2012. An Adaptive Model for Highway Travel Time Prediction, Journal of Advanced Transportation. DOI: http://dx.doi.org/10.1002/atr.1216, 48(6): 642-654.

Mahmoudabadi, A. 2010. Using Artificial Neural Network to Estimate Average Speed of Vehicles in Rural Roads. In Proceedings of the International Conference on Intelligent Networking and Computing.

Mazloumi, E.; Moridpour, S.; Currie, G.; Rose, G. 2012. Exploring the Value of Traffic Flow Data in Bus Travel Time Prediction, Journal of Transportation Engineering. DOI: http://dx.doi.org/10.1061/(ASCE)TE.19435436.0000329, 138(4): 436-446. 
Neural Ware Inc. 1993. Neural Computing. Technical Publications Group, Pittsburgh, PA.

Patnaik, J.; Chien, S.; Bladikas, A. 2004. Estimation of Bus Arrival Times Using APC Data, Journal of Public Transportation, 7(1): 1-20.

Ramakrishna, Y.; Ramakrishna, P.; Lakshmanan, V.; Sivanandan, R. 2006. Bus Travel Time Prediction using GPS Data. In Proceedings of the Map India. Available from Internet: <http://www.gisdevelopment.net/ proceedings/mapindia/2006/>.

Rice, J.; Zwet, V.E. 2004. A Simple and Effective Method for Predicting Travel Times on Freeways, IEEE Transactions on Intelligent Transportation Systems. DOI: http://dx.doi.org/10.1109/TITS.2004.833765, 5(3): 200-207.

Shalaby, A.; Farhan, A. 2004. Prediction Model of Bus Arrival and Departure Times Using AVL and APC Data, Journal of Public Transportation, 7(1): 41-61.

Sinha, K.C. 2003. Sustainability and Urban Public Transportation, Journal of Transportation Engineering, 1294: 331-341.

Sivanandam, S.N.; Sumathi, S.; Deepa, S.N. 2010. Introduction to Neural Networks using Matlab 6.0, Tata McGraw Hill, New Delhi.

Smith, B.L.; Demetsky, M.J. 1995. Short-term Traffic Flow Prediction: Neural Network Approach, Transportation Research Record, 1453: 98-104.

Tong, H.Y.; Hung, W.T. 2002. Neural Network Modelling of Vehicle Discharge Headway at Signalized Intersection: Model Description and Results, Transportation Research Part A: Policy and Practice. DOI: http://dx.doi.org/10.1016/S0965-8564(00)00035-5, 36(1): $17-40$.
Transport Research Board (TRB). 2001. Making Transit Work. Special Report, 257, National Academy, Washington, D.C.

Vanajakshi, L.; Subramanian, S.C.; Sivanandan, R. 2008. Travel Time Prediction under Heterogeneous Traffic condition using Global Positioning System Data from Buses, IET Intelligent Transport System. DOI: http://dx.doi. org/10.1049/iet-its:20080013, 3(1): 1-9.

Williams, B.; Hoel, L. 2003. Modelling and Forecasting Vehicle Traffic Flow as a Seasonal Arima Process: Theoretical Basis and Empirical Results, Journal of Transportation Engineering, 129(6): 664-672.

You, J.; Kim, T.J. 2000. Development and Evaluation of Hybrid Travel Time Forecasting Model, Transportation Research Part C: Emerging Technologies. DOI: http://dx.doi. org/10.1016/S0968-090X(00)00012-7, 8(1-6): 231-256.

Yu, B.; Lam, W.H.K.; Tam, M.L. 2011. Bus Travel Time Prediction at Bus Stop with Multiple Routes, Transportation Research Part C: Emerging Technologies. DOI: http://dx.doi.org/10.1016/j.trc.2011.01.003, 19(6): $1157-1170$.

Yu, B.; Yang, Z.-Z.; Chen, K.; Yu, B. 2010. Hybrid Model for Prediction of Bus Arrival Time at Next Station, Journal of Advanced Transportation. DOI: http://dx.doi. org/10.1002/atr.136, 44(3): 193-204.

Zhang, X.; Rice, J.A. 2003. Short-Term Travel Time Prediction Using A Time-Varying Coefficient Linear Model, Transportation Research Part C: Emerging Technologies, 11: 187-210.

Zheng, F.; Zuylen, H.V. 2013. Urban Link Travel Time Estimation Based on Sparse Probe Vehicle Data, Transportation Research Part C: Emerging Technologies. DOI: http://dx.doi.org/10.1016/j.trc.2012.04.007, 31: 145-147. 